\title{
Self-immolative systems for the disclosure of reactive electrophilic alkylating agents
}

\section{Article}

Published Version

Creative Commons: Attribution 4.0 (CC-BY)

Open access

Acton, A. L., Leroux, F., Feula, A., Melia, K., Sambrook, M. R., Hayes, W. and Russell, A. T. (2019) Self-immolative systems for the disclosure of reactive electrophilic alkylating agents. Chemical Communications, 55. pp. 5219-5222. ISSN 13597345 doi: https://doi.org/10.1039/C8CC09728C Available at https://centaur.reading.ac.uk/82743/

It is advisable to refer to the publisher's version if you intend to cite from the work. See Guidance on citing.

To link to this article DOI: http://dx.doi.org/10.1039/C8CC09728C

Publisher: The Royal Society of Chemistry

All outputs in CentAUR are protected by Intellectual Property Rights law, including copyright law. Copyright and IPR is retained by the creators or other copyright holders. Terms and conditions for use of this material are defined in the End User Agreement.

\section{www.reading.ac.uk/centaur}

\section{CentAUR}

Central Archive at the University of Reading 
Reading's research outputs online 


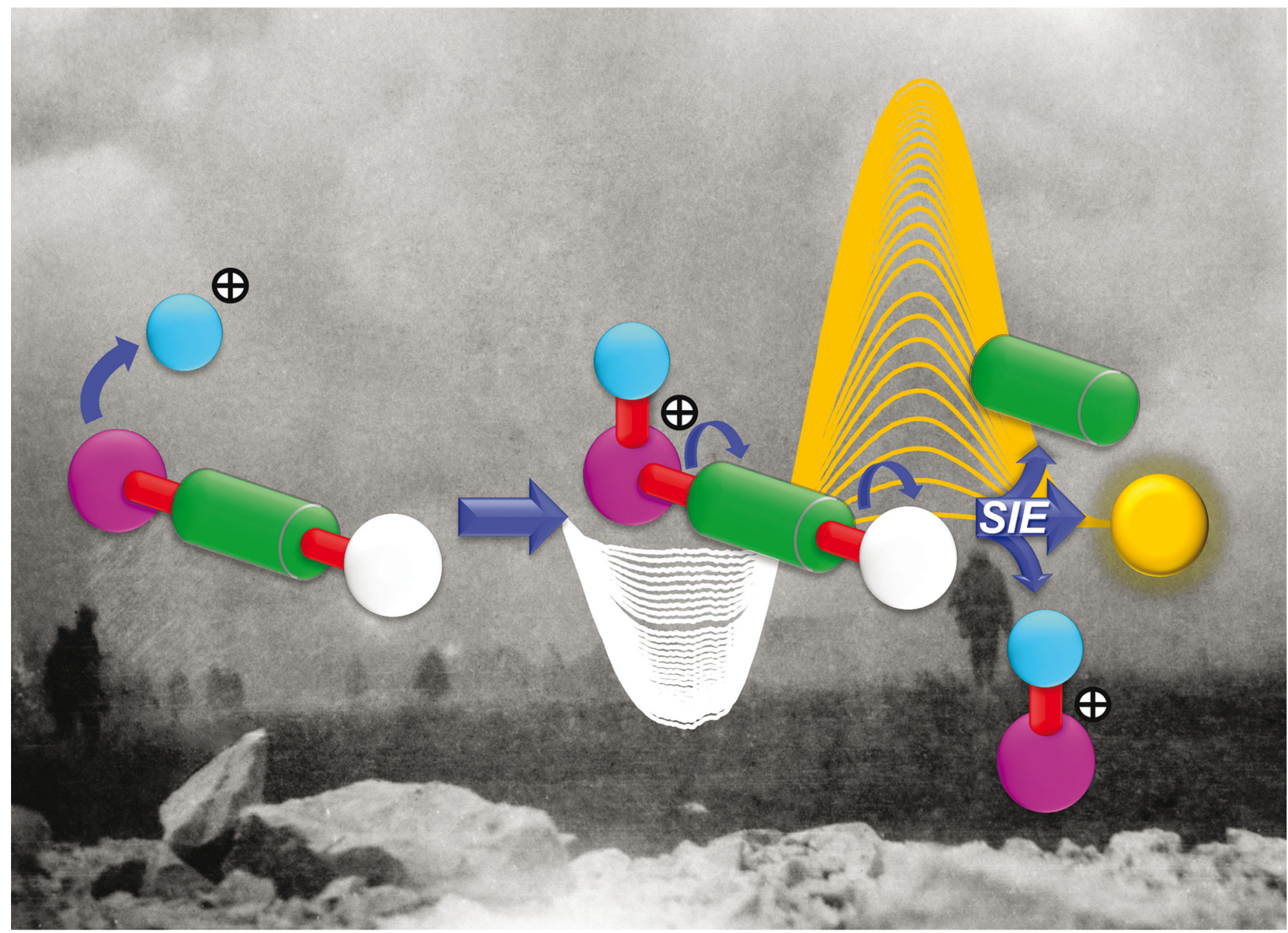

\section{Showcasing research from the School of Chemistry, University of Reading, Berkshire, UK.}

Self-immolative systems for the disclosure of reactive electrophilic alkylating agents

We report the synthesis and assessment of self-immolative systems triggered by non-acidic electrophiles such as methyl, allyl or benzylic halides. The systems provide a colorimetric response, upon exposure to these reagents under mild basic conditions, without need for analytical instrumentation.

Image credit: Imperial War Museum

\section{As featured in:}

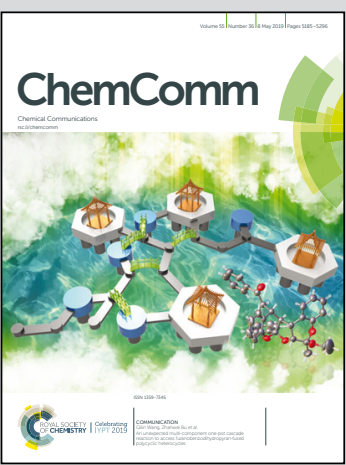

See Andrew T. Russell et al. Chem. Commun., 2019, 55, 5219. 
Check for updates

Cite this: Chem. Commun., 2019, 55,5219

Received 7th December 2018, Accepted 12th March 2019

DOI: $10.1039 / c 8 c c 09728 c$

rsc.li/chemcomm

In this paper we report the design, synthesis and assessment of the first examples of self-immolative systems triggered by non-acidic electrophilic agents such as methyl, allyl or benzylic halides. These systems provide a visual colorimetric disclosure response upon exposure to these electrophilic reagents under mild, basic conditions without the need for the use of analytical instrumentation.

The design of self-immolative systems ${ }^{1-3}$ has been based upon a stable bond between trigger and reporter groups ${ }^{4}$ the stability of which is sensitive to particular agents and environments (Scheme 1a). Chemically specific triggers have been realised for a diverse range of applications spanning drug delivery, ${ }^{5-7}$ biological and chemical sensors, ${ }^{8-10}$ diagnostics ${ }^{11}$ and degradable polymers. ${ }^{12-18}$ 'Classical' self-immolative chemistries operate by activation or deprotection of the trigger group by nucleophiles (e.g. fluoride, $\mathrm{RSH}$ ) to render the immolative unit labile via generation of an electron rich, nucleophilic centre that then initiates a cascade of electrons and culminates in the release of the reporter group (Scheme 1a). ${ }^{2-4}$ The obvious exception to this 'classical' approach to triggering of the self-immolative event is the electrophilic (protic) cleavage of a tert-butyloxycarbonyl (Boc) group to reveal, after neutralisation, a nucleophilic amine. ${ }^{19}$ This behaviour is peculiar to a proton; whereas, in the case of a general alkylating agent, reaction with a neutral nucleophile will generate a cationic species that is harder to convert to a nucleophile. To date, only limited use of self-immolative linkers in the disclosure of electrophilic species such as chemical warfare agents (CWAs), ${ }^{20,21}$ fumigants, pesticides, pharmaceuticals and other environmental

\footnotetext{
${ }^{a}$ Department of Chemistry, University of Reading, Whiteknights, Reading, RG6 6AD, UK. E-mail: a.t.russell@reading:ac.uk

${ }^{b}$ CBR Division, Defence Science \& Technology Laboratory (Dstl), Porton Down, Salisbury, Wiltshire, SP4 OJQ, UK

$\dagger$ Electronic supplementary information (ESI) available: Detailed experimental procedures and spectroscopic data for disclosure molecules 1-3. NMR spectra and kinetic curves for alkylation/ $\beta$-elimination reactions. NMR spectrum of the degradation study for the disclosure molecule 3. UV-visible spectrum for disclosure molecule 3 and UV-visible spectra for alkylation/ $\beta$-elimination reactions using disclosure molecule 3. See DOI: 10.1039/c8cc09728c
}

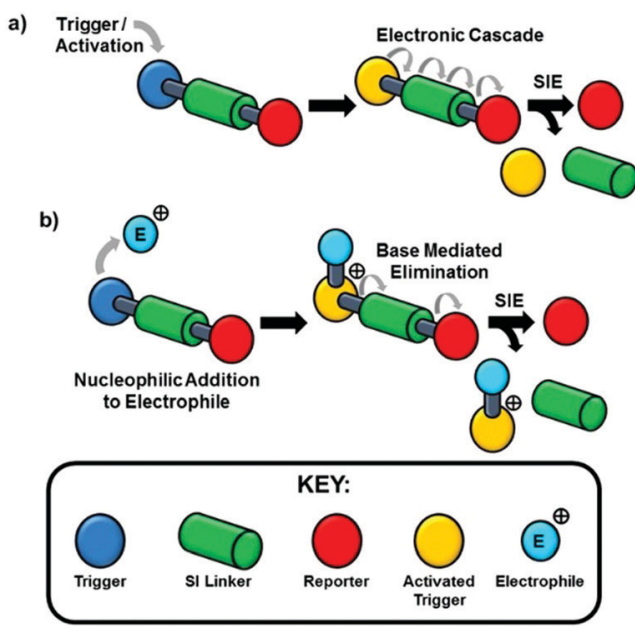

Scheme 1 (a) Schematic representation of a self-immolative system that becomes labile upon activation of a trigger group via the generation of an electron rich nucleophilic centre, in turn initiating a cascade of electrons and culminates in the release of a reporter group; (b) concept for a selfimmolative system for specific disclosure of electrophilic reagents.

hazards, has been reported and that by the vicarious generation of a nucleophile to trigger the self-immolative pathway. The challenge in generating a colorimetric disclosure system for reagents of this reactivity type under ambient conditions is to create a triggering system that responds to an electrophilic reagent in such a way as to allow indirect generation of a nucleophilic electron-rich centre in the trigger event (Scheme 1b).

In this communication, we report how we have met this challenge by designing a series of disclosure molecules (see 1-3 in Fig. 1) that represent the first examples of selective self-immolative systems triggered by a non-acidic electrophilic species such as methyl, allyl and benzylic halides as distinct from a proton, for the avoidance of false positive results. These systems provide a visual colorimetric response to such electrophiles, following exposure to mild, basic conditions, without the need for analytical instrumentation and thus there is great potential for the use of these reagents for the visual disclosure of toxic electrophilic alkylating agents. 


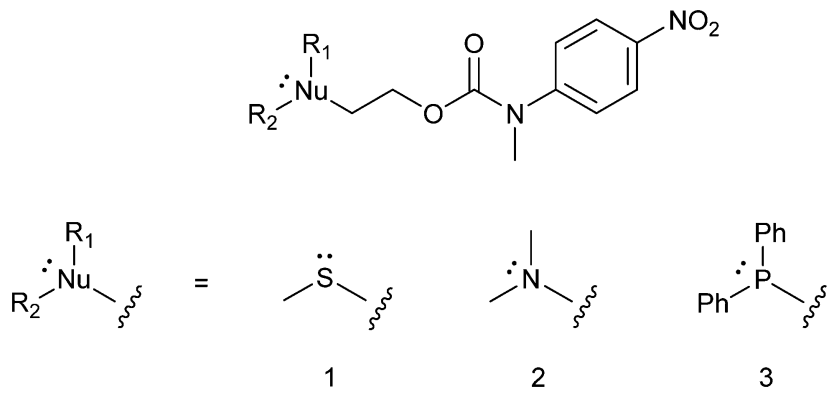

Fig. 1 The design of the self-immolative disclosure systems 1-3 for nonacidic electrophiles.

Based upon a published report by Crane and Rydon of studies on the reactions of mustard gas in biological systems, conducted during World War II at the Chemical Defence Experimental Station, Porton Down, UK, ${ }^{22}$ we based our design of the electrophile disclosure system on the known, but seldom employed urethane-based protecting group, 2-(methylthio)ethoxycarbonyl (Mteoc) that was developed by Kunz. ${ }^{23 a}$ Deprotection of the Mteoc group is traditionally facilitated in two steps via alkylation of the thioether group using methyl iodide (MeI) followed by the addition of base to promote $\beta$-elimination and liberation of the protected amine group..$^{23,24}$ The Mteoc group can be exploited as an electrophile labile trigger group and adapted to produce a series of potential molecular disclosure agents (1-3, Fig. 1) $)^{23 b}$ that upon exposure to an electrophile releases a coloured reporter group, for example, $N$-methyl-4-nitroaniline (Scheme 2). Electrophile disclosure is achieved via alkylation of the trigger group (at the $\mathrm{Nu}$ position) leading to an increase in the acidity of the $\alpha$-protons ${ }^{25}$ that in basic media results in self-immolative $\beta$-elimination with concomitant decarboxylation and release of the reporter group. The reporter group, when bound, is colourless but upon release a vibrant visual (yellow) colouration of the disclosure media is observed, thereby indicating the presence of the electrophile.

The effectiveness of disclosure systems 1-3 in providing a coloured response, upon exposure to electrophiles, is reliant on their rates of alkylation and elimination/decarboxylation. The intent to develop a system that could be deployed in real world scenarios, without use of instrumentation, mandated that it should work at room temperature and without the need for dry solvents. Thus, three different possible trigger groups were assessed, namely S, N and P-based derivatives (1-3 in Fig. 1),
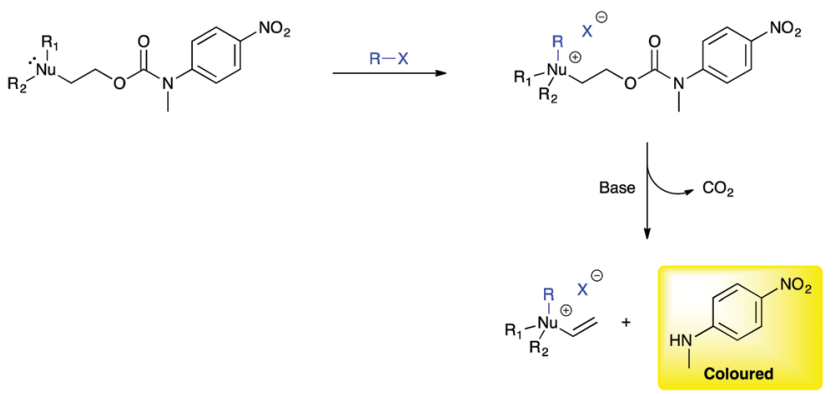

Scheme 2 The proposed self-immolative mechanism for the disclosure of electrophiles using 1-3 ( $\mathrm{Nu}=\mathrm{S}, \mathrm{N}, \mathrm{P}$ and $\mathrm{X}=\mathrm{I}, \mathrm{Br}, \mathrm{Cl})$.

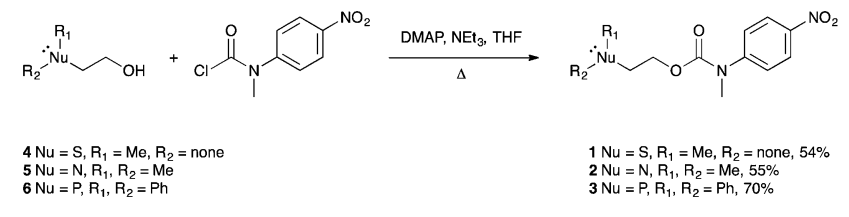

Scheme 3 Synthesis of the self-immolative disclosure systems 1-3.

in order to identify which of the trigger groups affords the optimum balance between the rate of alkylation and subsequent elimination, to release the reporter group, upon exposure to electrophiles in basic media. The rate of alkylation of nitrogen analogue 2 further served to assess whether it would be possible to use amines as bases in the presence of $\mathbf{1}$ and $\mathbf{3}$.

The target self-immolative systems were obtained via the conjugation of 2-(methylthio)ethanol 4, 2-dimethylaminoethanol 5 or 2-diphenylphosphinoethanol ${ }^{26} 6$ to $N$-methyl- $N$-(4-nitrophenyl)carbamoyl chloride ${ }^{27}$ using DMAP as a catalyst and heating under reflux in THF (Scheme 3, see Fig. S1-S12 in ESI $\dagger$ for the synthetic methods used and analytical data for 1-3).

Initial alkylation studies were conducted using the simple alkyl halide MeI. Cognizant that these are Type II $\mathrm{S}_{\mathrm{N}} 2$ reactions, ${ }^{28}$ alkylation of detectors 1-3 was conducted by dissolution of the detector in the polar aprotic solvent $\mathrm{CD}_{3} \mathrm{CN}$, followed by the addition of 10 molar equivalents of MeI and recording the ${ }^{1} \mathrm{H}$ NMR spectra at regular time intervals at $20{ }^{\circ} \mathrm{C}$. Fig. 2 displays example spectra obtained before and after the addition of MeI to a solution of 3 in $\mathrm{CD}_{3} \mathrm{CN}$ (for analogous spectroscopic studies on 1 and 2, see ESI, $\dagger$ Fig. S13 and S14).

The rate of alkylation was calculated by integration of the singlet resonance at $3.2 \mathrm{ppm}$ corresponding to the $N$-methyl group of 3 with respect to time. Alkylation was demonstrated to follow pseudo-first order kinetics and the half-life $\left(t_{1 / 2}\right)$ of alkylation of detector 3 with MeI was calculated to be 4.6 minutes (see ESI, $\dagger$ Fig. S15). The rate of alkylation for disclosure systems 1 and 2 were calculated using the corresponding ${ }^{1} \mathrm{H}$ NMR spectra in an
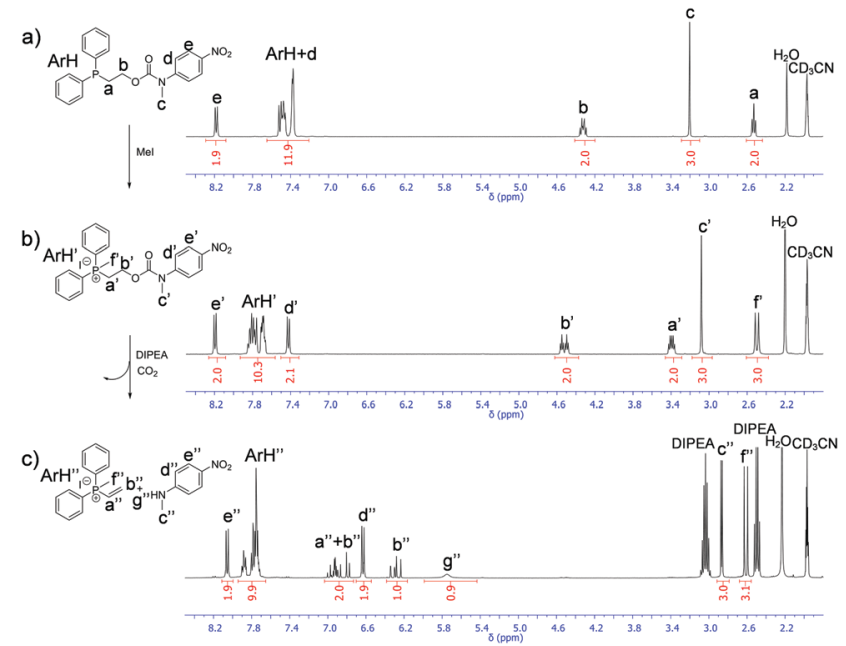

Fig. $2{ }^{1} \mathrm{H}$ NMR spectra obtained in $\mathrm{CD}_{3} \mathrm{CN}$ at $20{ }^{\circ} \mathrm{C}$ (a) before the addition, (b) 20 minutes after the addition of Mel to a solution of $\mathbf{3}$, (c) 1080 minutes after addition of DIPEA to a solution of alkylated $\mathbf{3}$. 
Table 1 Alkylation and elimination rate data obtained following the addition of 10 molar equivalents of Mel to a solution of disclosure systems 1-3 in $\mathrm{CD}_{3} \mathrm{CN}$ at $20{ }^{\circ} \mathrm{C}$ calculated using ${ }^{1} \mathrm{H}$ NMR spectroscopic data

\begin{tabular}{lll}
\hline $\begin{array}{l}\text { Disclosure } \\
\text { system }\end{array}$ & $\begin{array}{l}\text { MeI alkylation } \\
t_{1 / 2} \text { (minutes) }\end{array}$ & $\begin{array}{l}\beta \text {-Elimination } \\
t_{1 / 2} \text { (minutes) }\end{array}$ \\
\hline 1 & $1994^{a}$ & $>900$ \\
2 & 1.4 & None \\
3 & 4.6 & 120
\end{tabular}

${ }^{a}$ Methyl iodide does not follow pseudo-first order kinetics, $t_{1 / 2}$ is taken as the time taken for $50 \%$ of disclosure system 1 to be alkylated (see ESI, Fig. S16). The alkylation of $\mathbf{1}$ can be significantly accelerated by the addition of $\mathrm{AgSbF}_{6}$ and the reaction becomes pseudo-first order (see ESI, Fig. S16 and S38). However, the precipitation of silver salts renders the colour change of the self-immolative system, following elimination, less clear. ${ }^{31}$

analogous manner and the half-lives of alkylation are shown in Table 1 . The methylated products of $\mathbf{1}$ and $\mathbf{3}$ undergo elimination when $N, N$-diisopropylethylamine (DIPEA) is added to release the reporter unit, $N$-methyl-4-nitroaniline (Fig. 2c); their half-lives are recorded in Table 1 . The best balance between the rates of alkylation and elimination was achieved with the phosphine disclosure system 3 . Though alkylation of 2 was found to be slightly faster than $\mathbf{3}$, it was postulated that a bulky amine base could still work if included in a one-pot alkylation-elimination mixture (vide infra).

The rates of alkylation and elimination were then investigated using disclosure system 3 with different alkylating agents, as shown in Table 2. For most of the alkylating agents, rates could be increased significantly by nucleophilic catalysis with sodium iodide; a Finkelstein displacement presumably preceding alkylation of detector 3. Only in the case of benzyl chloride and allyl chloride, the alkylation proved to be slow and did not follow pseudo-first order kinetics (see ESI, $\dagger$ Fig. S17).

Disclosure systems for electrophilic reagents must be capable of being used in a real world environment without generating

Table 2 Alkylation rate data obtained following the addition of 10 molar equivalents of different alkylating agents, e.g. benzyl halides, xylyl bromides, allyl halides, trityl chloride and 10 molar equivalents of $\mathrm{Nal}$ to a solution of disclosure system 3 in $\mathrm{CD}_{3} \mathrm{CN}$ at $20{ }^{\circ} \mathrm{C}$ calculated using ${ }^{1} \mathrm{H}$ NMR spectroscopic data

\begin{tabular}{|c|c|c|c|}
\hline Alkylating agent & $\begin{array}{l}\text { Alkylation } \\
t_{1 / 2} \text { (minutes) }\end{array}$ & $\begin{array}{l}\text { Alkylation with } \\
\mathrm{NaI} t_{1 / 2} \text { (minutes) }\end{array}$ & $\begin{array}{l}\beta \text {-Elimination } \\
t_{1 / 2} \text { (minutes) }\end{array}$ \\
\hline Methyl iodide & 4.6 & - & 120 \\
\hline Benzyl bromide & 14.5 & $<5$ & 40 \\
\hline Benzyl chloride & $>2000$ & $24^{a}$ & $1050^{b}$ \\
\hline $\begin{array}{l}\text { 2-Methylbenzyl } \\
\text { bromide }\end{array}$ & 4.4 & $<5$ & 60 \\
\hline $\begin{array}{l}\text { 3-Methylbenzyl } \\
\text { bromide }\end{array}$ & 9.5 & $<5$ & 33 \\
\hline $\begin{array}{l}\text { 4-Methylbenzyl } \\
\text { bromide }\end{array}$ & 9.6 & $<5$ & 47 \\
\hline Allyl bromide & 77 & 10.7 & 116 \\
\hline Allyl chloride & $>2000$ & $96^{a}$ & $570^{b}$ \\
\hline Trityl chloride & 113.6 & $-^{c}$ & 55 \\
\hline
\end{tabular}

${ }^{a}$ Benzyl/allyl chloride does not follow pseudo-first order kinetics, $t_{1 / 2}$ is taken as the time taken for $50 \%$ of disclosure system 3 to alkylate. ${ }^{b}$ Side reaction of $N$-alkylation with DIPEA occurred, slowing down the rate of $\beta$-elimination (see ESI, Fig. S18 and S19). ${ }^{c}$ Under these conditions, alkylation was not observed. false positives and thus must be stable to environmental moisture (in this case, to avoid adventitious hydrolysis of the urethane group). Disclosure system 3 has proved to be successful in the disclosure of alkylating agents, but its stability in aqueous media required assessment. Therefore, degradation studies were performed using NMR spectroscopy and a solvent mixture of $\mathrm{CD}_{3} \mathrm{CN}$ with $10 \%$ of $\mathrm{D}_{2} \mathrm{O}$. After exposure of disclosure system 3 to $\mathrm{CD}_{3} \mathrm{CN}$ with $10 \%$ of $\mathrm{D}_{2} \mathrm{O}$, no significant degradation was observed in the ${ }^{1} \mathrm{H}$ NMR spectrum after 20 hours (see ESI, $\dagger$ Fig. S20), indicating its potential stability toward environmental moisture.

In this case, to obtain an efficient disclosure system, it is important that all the required reagents can be present simultaneously and that the alkylation and elimination reactions can occur sequentially whilst avoiding side reactions. Thus, the one-pot reaction of alkylation-elimination was carried out by mixing the disclosure system 3 with two molar equivalents of DIPEA before addition of the alkylating agent. ${ }^{29}$

It can be seen that, after addition of 10 molar equivalents of benzyl bromide, the disclosure system 3 was alkylated and this intermediate undergoes elimination to produce the reporter group, $\mathrm{N}$-methyl-4-nitroaniline (Fig. 3), demonstrating the onepot alkylation-elimination process. Furthermore, the strong yellow colouration due to the release of $\mathrm{N}$-methyl-4-nitroaniline

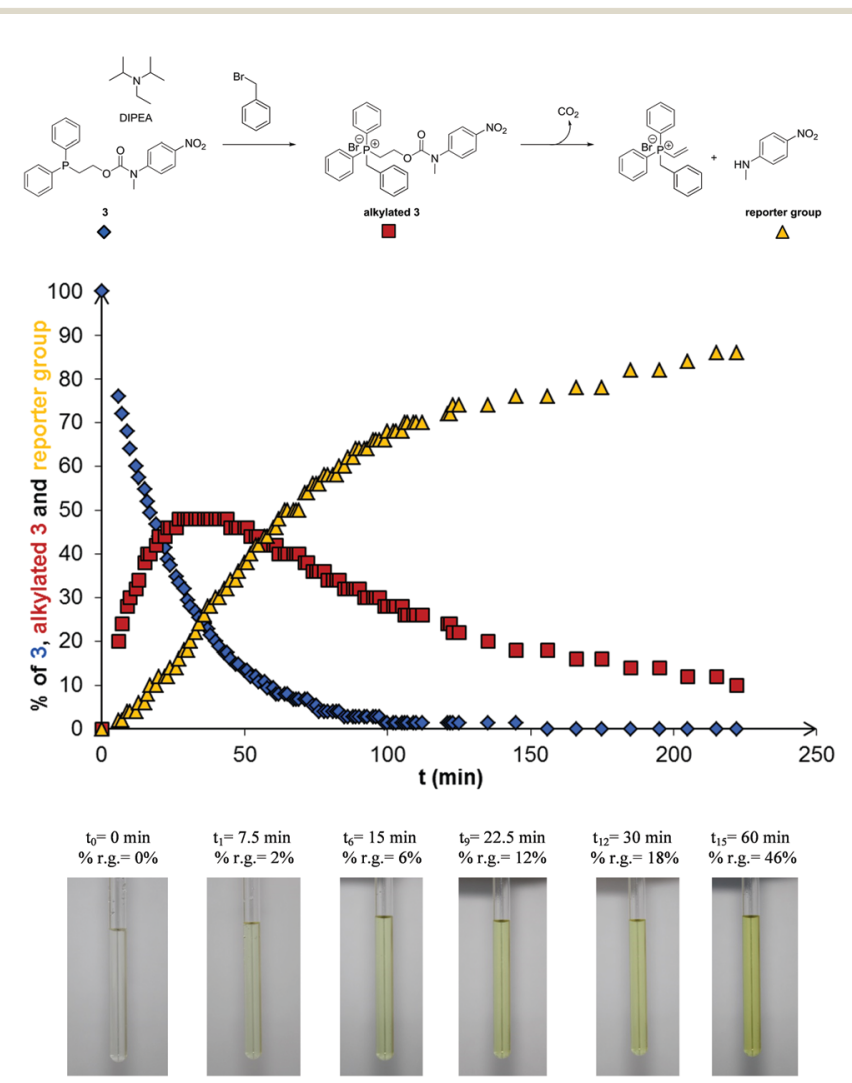

Fig. 3 Top: Reaction schematic of self-immolative degradation of disclosure system 3. Middle: Reaction kinetics calculated from the ${ }^{1} \mathrm{H}$ NMR spectra obtained following the addition of 10 molar equivalents of benzyl bromide to 3 and DIPEA. Bottom: Images of the NMR tube containing the solution of $\mathbf{3}\left([3]_{0}=2.5 \times 10^{-2} \mathrm{~mol} \mathrm{~L}^{-1}\right)$ before addition of benzyl bromide and $7.5,15,22.5,30$ and 60 minutes after addition of benzyl bromide, respectively. 
was observed in significantly less than 10 minutes after addition of the DIPEA (see ESI, $\dagger$ Fig. S21).

The ability to disclose at lower concentrations of alkylating agents using 3 was investigated with benzyl bromide as an alkylating agent by decreasing, in the first instance, the ratio of benzyl bromide to 3 to $1.85 / 1$ ([benzyl bromide $]_{0}=0.046 \mathrm{~mol} \mathrm{~L}^{-1}$ ). Alkylation of 3 was demonstrated to follow second order kinetics with a half-life $\left(t_{1 / 2}\right)$ calculated to be 145 minutes (see ESI, $\dagger$ Fig. S22-S24 and Table S1). The release and concentration of reporter group $\mathrm{N}$-methyl-4-nitroaniline was then monitored by UV-visible spectroscopy from a solution of alkylated 3 in relation to an external calibration plot (see ESI, $\dagger$ Fig. S25-S27). In an analogous one-pot study, the concentration of both 3 and benzyl bromide were decreased 10 -fold and the release of the reporter group monitored with respect to time. The concentration of the reporter group was observed to increase after addition of benzyl bromide and clear visual disclosure was evidenced after 300 minutes (see ESI, $\dagger$ Fig. S28).

In this communication are reported the first examples of selective self-immolative systems that are triggered by reactive non-acidic electrophilic species such as methyl and benzylic halides.§ These self-immolative molecules successfully generate a distinct colorimetric response to non-acidic electrophiles following a designed two-step process that involves initial alkylation of the self-immolative unit followed by elimination via an in situ base. These self-immolative molecules could offer a practical route for the selective disclosure of electrophilic species, such as CWAs, outside of the laboratory environment and without need for instrumentation. Further studies are currently under way in order to produce clear and robust disclosure events for a broader range of electrophiles using alternative reporter groups.

This study was supported by Dstl (contracts DSTLX1000070800, DSTLX1000114599 and DSTLX1000097453, post-doctoral research fellowships for A. L. A., F. L., A. F. and K. M.). We thank the University of Reading for access to resources in the Chemical Analysis Facility.

\section{Conflicts of interest}

There are no conflicts to declare.

\section{Notes and references}

\$ The alkene region of (c) is not a simple, first order pattern, in contrast to the corresponding sulfur analogue (see ESI, $\dagger$ Fig. S29). ${ }^{30}$ However, detailed analysis of the spectroscopic data confirmed the structure of the vinyl phosphonium salt (see ESI, $\dagger$ Fig. S30-S37).

$\S$ Detailed spectroscopic studies on these self-immolative systems are reported in the ESI, $\dagger$ (see Fig. S39-S67 and Table S2).

1 P. L. Carl, P. K. Chakravarty and J. A. Katzenellenbogen, J. Med. Chem., 1981, 24, 479.

2 M. E. Roth, O. Green, S. Gnaim and D. Shabat, Chem. Rev., 2016, 116, 1309.

3 G. I. Peterson, M. B. Larsen and A. J. Boydston, Macromolecules, $2012,45,7317$.

4 C. A. Blencowe, A. T. Russell, F. Greco, W. Hayes and D. W. Thornthwaite, Polym. Chem., 2011, 2, 773.

5 I. Tranoy-Opalinski, A. Fernandes, M. Thomas, J. P. Gesson and S. Papot, Anti-Cancer Agents Med. Chem., 2008, 8, 618.

6 A. Sagi, E. Segal, R. Satchi-Fainaro and D. Shabat, Bioorg. Med. Chem., 2007, 15, 3720.

7 F. Kratz, I. A. Muller, C. Ryppa and A. Warnecke, ChemMedChem, 2008, 3, 20.

8 N. H. Ho, R. Weissleder and C. H. Tung, ChemBioChem, 2007, 8, 560.

9 M. Avital-Shmilovici and D. Shabat, Bioorg. Med. Chem. Lett., 2009, 19, 3959.

10 A. Sagi, R. Weinstain, N. Karton and D. Shabat, J. Am. Chem. Soc., 2008, 130, 5434.

11 E. Sella and D. Shabat, J. Am. Chem. Soc., 2009, 131, 9934.

12 A. Sagi, R. Weinstain, N. Karton and D. Shabat, J. Am. Chem. Soc., 2008, 130, 5434.

13 R. Weinstain, A. Sagi, N. Karton and D. Shabat, Chem. - Eur. J., 2008, 14, 6857.

14 M. A. DeWit and E. R. Gillies, J. Am. Chem. Soc., 2009, 131, 18327.

15 A. P. Esser-Kahn, N. R. Sottos, S. R. White and J. S. Moore, J. Am. Chem. Soc., 2010, 132, 10266.

16 W. Seo and S. T. Phillips, J. Am. Chem. Soc., 2010, 132, 9234.

17 A. M. DiLauro, G. G. Lewis and S. T. Phillips, Angew. Chem., Int. Ed., 2015, 127, 6298.

18 S. Gnaim and D. Shabat, Acc. Chem. Res., 2014, 47, 2970.

19 M. Shamis and D. Shabat, Chem. - Eur. J., 2007, 13, 4523.

20 X. Sun, J. F. Reuther, S. T. Phillips and E. V. Anslyn, Chem. - Eur. J., 2017, 23, 3903.

21 X. Sun, A. A. Boulgakov, L. N. Smith, P. Metola, E. M. Marcotte and E. V. Anslyn, ACS Cent. Sci., 2018, 4, 854.

22 C. W. Crane and H. N. Rydon, J. Chem. Soc., 1947, 766.

23 (a) H. Kunz, Chem. Ber., 1976, 109, 3693; (b) D. Chantreux, J.-P. Gamet, R. Jacquier and J. Verducci, Tetrahedron, 1984, 40, 3087.

24 R. Barthels and H. Kunz, Z. Naturforsch., B: Anorg. Chem., Org. Chem., 1979, 34, 1121.

25 F. Bernardi, H. B. Schlegel, M.-H. Whangbo and S. Wolfe, J. Am. Chem. Soc., 1977, 99, 5633.

26 N. Kanbayashi and K. Onitsuka, Angew. Chem., Int. Ed., 2011, 50, 5197.

27 T. Iwai, T. Fujihara, J. Terao and Y. Tsuji, J. Am. Chem. Soc., 2010, 132, 9602.

28 (a) E. D. Hughes and C. K. Ingold, J. Chem. Soc., 1935, 244; (b) C. K. Ingold, Structure and Mechanism in Organic Chemistry, G. Bell and Sons Ltd, London, 1953, p. 346.

29 J. L. Moore, S. M. Taylor and V. A. Soloshonok, ARKIVOC, 2005, vi, 287.

30 M. Yar, E. M. McGarrigle and V. K. Aggarwal, Angew. Chem., Int. Ed., 2008, 47, 3784.

31 K. Hojo, M. Maeda and K. Kawasaki, Tetrahedron, 2004, 60, 1875. 